\title{
NG2, a novel proapoptotic receptor, opposes integrin $\alpha 4$ to mediate anoikis through PKC $\alpha$-dependent suppression of FAK phosphorylation
}

\author{
NE Joo ${ }^{1}, T_{\text {Watanabe }}{ }^{1}, C$ Chen ${ }^{1}$, M Chekenya ${ }^{2}$, WB Stallcup ${ }^{3}$ and YL Kapila ${ }^{*, 1}$
}

Disruption of cell-matrix interactions can lead to anoikis - apoptosis due to loss of matrix contacts. Altered fibronectin (FN) induces anoikis of primary human fibroblasts by a novel signaling pathway characterized by reduced phosphorylation of focal adhesion kinase (FAK). However, the receptors involved are unknown. FAK phosphorylation is regulated by nerve/glial antigen 2 (NG2) receptor signaling through $\mathrm{PKC} \alpha$ a point at which signals from integrins and proteoglycans may converge. We found that an altered FN matrix induced anoikis in fibroblasts by upregulating NG2 and downregulating integrin $\alpha 4$. Suppressing NG2 expression or overexpressing $\alpha 4$ rescued cells from anoikis. NG2 overexpression alone induced apoptosis and, by reducing FAK phosphorylation, increased anoikis induced by an altered matrix. NG2 overexpression or an altered matrix also suppressed PKC $\alpha$ expression, but overexpressing integrin $\alpha 4$ enhanced FAK phosphorylation independently of PKC $\alpha$. Cotransfection with NG2 CDNA and integrin $\alpha 4$ siRNA did not lower PKC $\alpha$ and pFAK levels more than transfection with either alone. PKC $\alpha$ was upstream of FAK phosphorylation, as silencing PKC $\alpha$ decreased FAK phosphorylation. PKC $\alpha$ overexpression reversed this behavior and rescued cells from anoikis. Thus, NG2 is a novel proapoptotic receptor, and NG2 and integrin $\alpha 4$ oppositely regulate anoikis in fibroblasts. NG2 and integrin $\alpha 4$ regulate FAK phosphorylation by PKC $\alpha$-dependent and -independent pathways, respectively.

Cell Death and Differentiation (2008) 15, 899-907; doi:10.1038/cdd.2008.22; published online 22 February 2008

The extracellular matrix (ECM) glycoprotein fibronectin (FN) affects adhesion, migration, survival, and other cellular functions. FN fragments found in vivo and associated with disease or comparable recombinant fragments trigger apoptosis/anoikis in primary human fibroblasts via a novel pathway regulated by decreases in focal adhesion kinase (FAK) phosphorylation and downregulation of p53. ${ }^{1-3}$ However, the receptors involved are not known. Two classes of cellsurface receptors, integrins and proteoglycans, interact with $\mathrm{FN}$ and could mediate the anoikis. This process is regulated by FAK, an integrin-dependent non-receptor tyrosine kinase, consistent with a role for integrins. Also proteoglycans are involved in this apoptotic mechanism. ${ }^{1}$

Integrins are $\alpha$ and $\beta$ heterodimeric cell-surface receptors that mediate cell-ECM interactions and regulate cell adhesion, migration, proliferation, and survival. Interactions between $\mathrm{FN}$ and integrins promote the survival of many cell types. We showed that blocking antibodies against the $\alpha 4$ integrin subunit induced apoptosis-like cell rounding in human fibroblasts, similar to that induced by anoikis in response to an altered FN matrix fragment. ${ }^{2}$ Integrins mediate those responses by binding ECM ligands and activating signaling cascades that promote these actions. The $\alpha 4$ integrin subunit can bind to at least three interaction sites on FN. These include the arginine-glycine-aspartic acid site on the central cell-binding domain, the $\mathrm{V}$ region, and the heparin-binding domain. Once engaged, integrins alter cell behavior by recruiting and activating signaling proteins, including FAK. Integrin-FAK signaling complexes may help regulate anchorage-dependent cell survival. ${ }^{4}$ FAK is critical in protecting cells from apoptosis, but the mechanisms are not fully characterized. ${ }^{4,5}$ Constitutive activation of FAK protects epithelial cells from anoikis, ${ }^{4}$ while interference with FAK function in fibroblasts and endothelial cells triggers apoptosis by a p53-dependent mechanism. ${ }^{5,6} \mathrm{PKC}$ also plays a role in integrin-mediated cell adhesion, spreading, ${ }^{7,8}$ migration, ${ }^{7}$ FAK phosphorylation, ${ }^{9}$ and focal adhesion formation. ${ }^{10}$ PKC appears to be a key intermediate between integrins and FAK signaling in many cell types. ${ }^{11} \mathrm{PKC}$ activation is required for FAK phosphorylation in cells plated on $\mathrm{FN}^{7,9}$

FN also interacts with proteoglycans. Proteoglycans have been implicated in the regulation of apoptotic pathways, but little is known about the mechanism. The chondroitin sulfate proteoglycan (CSPG) nerve/glial antigen 2 (NG2) and the $\alpha 4 \beta 1$ integrin have been proposed as coreceptors with distinct signaling roles in mediating the spread of melanoma cells on FN-coated surfaces. ${ }^{12,13}$ Thus, NG2 might work in concert with $\alpha 4 \beta 1$ to regulate anoikis induced by an altered FN matrix.

\footnotetext{
${ }^{1}$ Department of Periodontics and Oral Medicine, School of Dentistry, University of Michigan, Ann Arbor, MI, USA; ${ }^{2}$ Norlux Neuro-Oncology, Department of Biomedicine, University of Bergen, Bergen, Norway and ${ }^{3}$ Cancer Research Center, The Burnham Institute for Medical Research, La Jolla, CA, USA

${ }^{*}$ Corresponding author: YL Kapila, Department of Periodontics and Oral Medicine, School of Dentistry, University of Michigan, 1101 N University Avenue, Ann Arbor, MI 48109-1078, USA. Tel: + 734615 2295; Fax: + 734763 5503; E-mail: ykapila@umich.edu

Keywords: altered FN matrix; anoikis; FAK; integrin $\alpha 4$; NG2; PKC $\alpha$

Abbreviations: ECM, extracellular matrix; FAK, focal adhesion kinase; FN, fibronectin; NG2, nerve/glial antigen 2

Received 13.9.07; revised 16.1.08; accepted 21.1.08; Edited by H Ichijo; published online 22.2.08
} 
NG2 is expressed on the surface of various cell types and its expression decreases with terminal differentiation. ${ }^{14,15}$ NG2 is a transmembrane receptor that interacts with ECM molecules, such as type VI collagen, and with other cellsurface components, including $\beta 1$ integrins, to mediate cell adhesion and proliferation. ${ }^{16,17}$ The cytoplasmic domain of NG2 interacts with scaffolding proteins such as MUPP $1^{18}$ and GRIP1 $1^{19}$ and with kinases such as PKC $\alpha$ and ERK.${ }^{17}$ NG2 also regulates $\mathrm{FAK}$ and $\mathrm{ERK}$ activation by distinct mechanisms in human melanoma cells. ${ }^{13}$ However, regulation of FAK phosphorylation through proteoglycans in apoptosis has not been studied, and the role of NG2 in regulating apoptosis by the ECM is unknown.

We tested the hypothesis that NG2 acts in concert with $\alpha 4 \beta 1$ to regulate anoikis induced by an altered $F N$ matrix $(\mathrm{V}+\mathrm{H}-)$ in human fibroblasts. We also tested the possibility that $\mathrm{PKC} \alpha$ and FAK signaling are control points where these two receptor-mediated signaling pathways converge to regulate this process.

\section{Results}

A CSPG is involved in anoikis triggered by an altered FN matrix. As previously shown, ${ }^{1-3}$ an altered FN matrix consisting of the $\mathrm{V}+\mathrm{H}$ - protein induced apoptosis in primary ligament fibroblasts (Figure 1a and b). Cells treated with an altered FN matrix exhibited nuclear condensation and bright DAPI staining typical of apoptotic cells. Cells treated with an altered FN matrix also had significantly higher levels of apoptosis, as assessed by flow cytometry, than cells treated with serum-free medium or the control $\mathrm{V}+\mathrm{H}+\mathrm{FN}$ protein.

To identify the receptors mediating this apoptotic mechanism, we first determined if proteoglycans might be involved, as FN binds to proteoglycans and integrins. Specifically, since the heparin-binding domain of FN interacts with CSPGs and heparin sulfate proteoglycans, we investigated this possibility under anoikis conditions by altering surface GAGs, using the traditional method of removing $\mathrm{CS}$ and/or HS with specific enzymes. ${ }^{1}$ Pretreatment with chondroitinase $A B C$ rescued cells from apoptosis induced by the altered FN matrix (Figure 1c), but pretreatment with heparinase III did not (Figure 1d). Cell rescue after pretreatment with both glycosaminoglycans was similar to that in cells treated with chondroitinase $\mathrm{ABC}$ alone (Figure 1e).

NG2 proteoglycan expression is regulated by anoikis. To determine if a CSPG receptor mediates anoikis triggered by an altered FN matrix, we measured the expression of several CSPGs. In cells treated with an altered FN matrix, NG2 levels increased at $2 \mathrm{~h}$ and decreased at $4 \mathrm{~h}$ (Figure 2a), as shown by western blotting; CD44, CD44s, syndecan-2, and syndecan-4 levels were unchanged (Figure 2b). These findings were confirmed by flow cytometric and immunofluorescence analysis (Figure 2c and d). As shown by RT-PCR, treatment with an altered FN matrix upregulated NG2 transcripts at $2 \mathrm{~h}$, but the downregulation at $4 \mathrm{~h}$ was not statistically significant (Figure 2e).
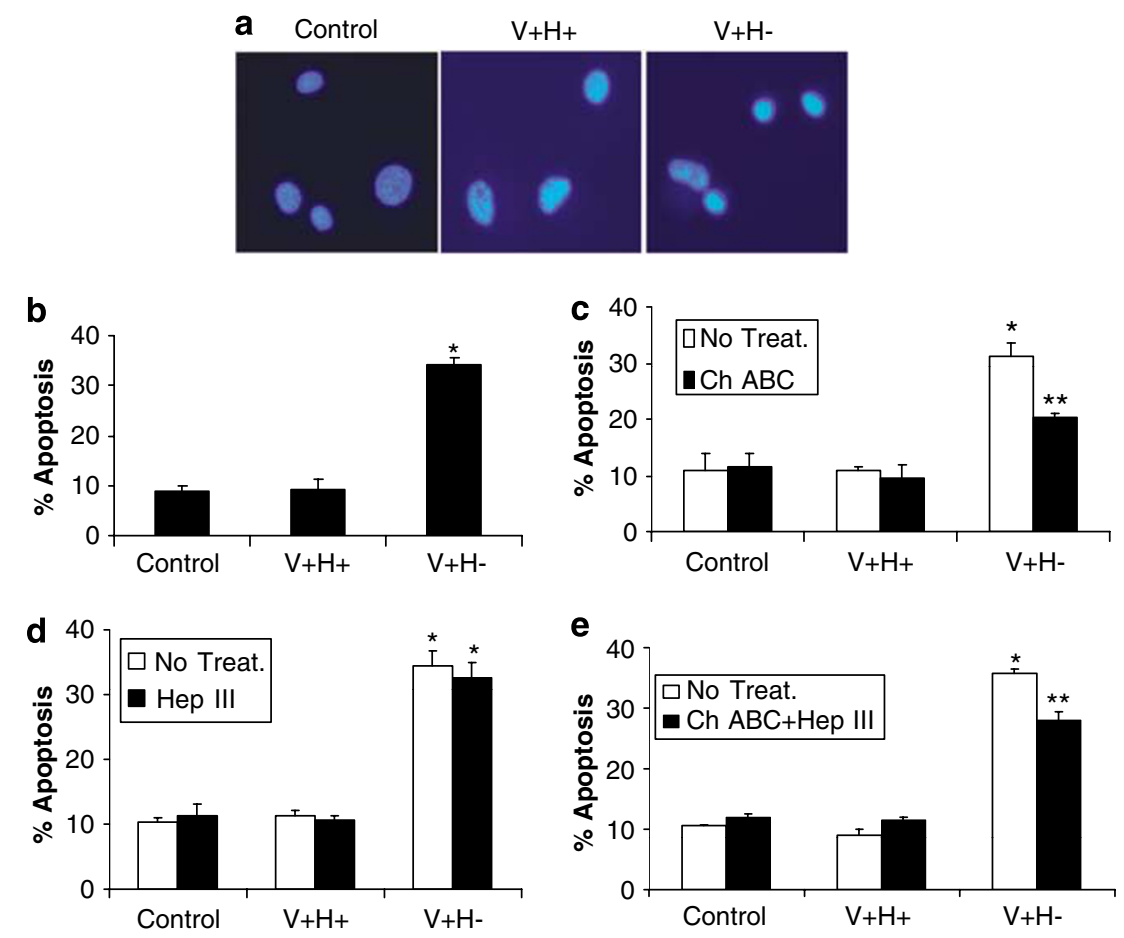

Figure 1 Induction of anoikis by an altered FN matrix in human primary fibroblasts depends on a CSPG. (a) Nuclear morphology of DAPI-stained cells treated with control serum-free medium (control) or medium supplemented with $\mathrm{V}+\mathrm{H}+$ or $\mathrm{V}+\mathrm{H}-\mathrm{FN}$ protein $(40 \mu \mathrm{g} / \mathrm{ml})$ for $4 \mathrm{~h}$. (b) Flow cytometric analysis of apoptotic cells treated as in (a). Flow cytometric analysis of apoptotic cells pretreated with chondroitinase ABC (Ch ABC) (c), heparinase III (Hep III) (d), or chondroitinase ABC and heparinase III (ChABC + Hep III) (e) for $1 \mathrm{~h}$ and treated with recombinant FN proteins as in (a). Values are mean \pm S.E.M. ${ }^{*} P<0.05$ versus control. ${ }^{\star \star} P<0.05$ versus $\mathrm{V}+\mathrm{H}-$ alone 

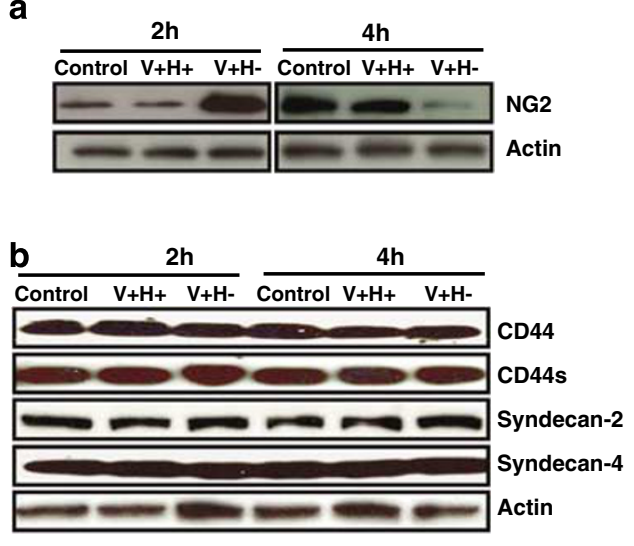
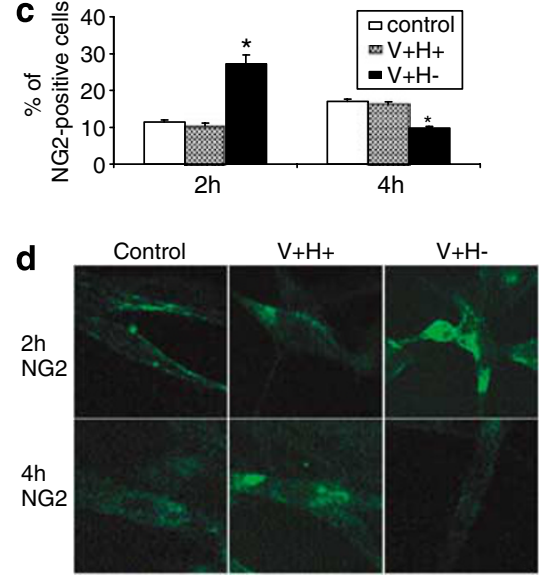

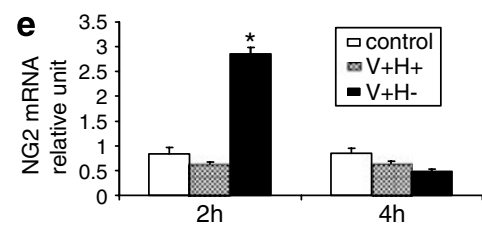

Figure 2 NG2 expression is first upregulated then downregulated under matrix conditions that induce anoikis in primary human fibroblasts. (a) Western blot of NG2 in cells treated with serum-free medium (control) or V $+\mathrm{H}+$ or $\mathrm{V}+\mathrm{H}-\mathrm{FN}$ protein $(40 \mu \mathrm{g} / \mathrm{ml})$ for 2 and $4 \mathrm{~h}$. (b) Western blot analysis of CD44, CD44s, syndecan-2, and syndecan-4 in cells treated as in (a). Actin served as a loading control. (c) Flow cytometric analysis of NG2 expression in cells treated as in (a). (d) Immunofluorescence analysis of NG2 expression in cells treated as in (a). Original magnification, $\times 200$. (e) Relative level of NG2 mRNA expression in cells treated as in (a). Values are mean \pm S.E.M. ${ }^{*} P<0.05$ versus control

NG2 is a proapoptotic receptor. Next, we determined if up- or downregulation of NG2 is critical for anoikis induced by an altered FN matrix. Transient transfection with NG2 siRNA $(100 \mathrm{pmol})$ rescued cells from apoptosis, as shown by morphology, a quantitative flow cytometric apoptosis assay, and a cell death ELISA assay that measures DNA fragmentation; however, transfection with NG2 cDNA $(1 \mu \mathrm{g})$ increased apoptosis, indicating that NG2 is a proapoptotic receptor (Figure 3a-e). The B5 antibody used for immunoblotting recognizes both endogenous human NG2 and transfected rat NG2, so the immunoblotting results represent the total amount of NG2 in the cells. These data also demonstrate that anoikis induced by the altered FN matrix is mediated by the increase in NG2 expression at $2 \mathrm{~h}$, not the decrease at $4 \mathrm{~h}$ (Figure $2 \mathrm{a}, \mathrm{c}-\mathrm{e}$ ). To further confirm that NG2 is a proapoptotic receptor, NG2-null (NG2-/-) cells were compared to NG2-positive (NG2+/+) cells under anoikis conditions. Like primary cells overexpressing NG2 siRNA, NG2-null cells were resistant to apoptosis (Figure $3 f-h$ ).

Anoikis conditions decrease integrin $\alpha \mathbf{4}$ expression. Since integrin $\alpha 4$ and the NG2 work cooperatively to mediate the spreading of melanoma cells on $\mathrm{FN},{ }^{12,13}$ we tested whether integrin $\alpha 4$ cooperates with NG2 to regulate anoikis in cells treated with an altered FN matrix. At 2 and $4 \mathrm{~h}$ after treatment, integrin $\alpha 4$ protein levels were decreased (Figure $4 a$ ), but integrin $\alpha 5$ and $\alpha \mathrm{v}$ levels were not affected (Figure $4 \mathrm{~b}$ ). These findings were confirmed by flow cytometric and immunofluorescence analyses (Figure $4 \mathrm{c}$ and $\mathrm{d}$ ). As shown by PCR analyses, integrin $\alpha 4$ mRNA levels trended lower in cells treated with an altered FN matrix, but the difference was not statistically significant (Figure 4e).
Integrin $\alpha \mathbf{4}$ is an anti-anoikis regulator. To further examine the role of integrin $\alpha 4$ in anoikis in primary fibroblasts, we overexpressed integrin $\alpha 4$ using cDNA and suppressed $\alpha 4$ expression with siRNA. Cells overexpressing integrin $\alpha 4$ and treated with altered FN matrix were well spread and were morphologically similar to transfection controls and cells treated with the control FN protein (Figure 5a). As shown by a quantitative flow cytometric assay and cell death ELISA assay, overexpression of integrin $\alpha 4$ rescued cells from apoptosis (Figure $5 b$ and $d$ ). Cells transfected with $\alpha 4$ siRNA were also morphologically similar to controls (Figure 5a). However, when they were treated with an altered FN matrix, apoptosis was more pronounced than in untransfected cells treated with altered FN matrix (Figure 5c and e), suggesting a synergistic effect of $\alpha 4$ siRNA. Interestingly, suppression of integrin $\alpha 4$ expression alone did not significantly affect apoptosis levels, compared to control transfected cells. Since basal levels of $\alpha 4$ are generally low in these cells (Figure $5 b$ ), western blots to detect $\alpha 4$ suppression by siRNA (Figure $5 \mathrm{c}$ ) were developed for a longer time.

FAK phosphorylation by NG2, but not by integrin $\alpha \mathbf{4}$, is $P K C \alpha$ dependent. To study the signaling mechanisms and potential cross-talk by which NG2 and integrin $\alpha 4$ regulate anoikis, we measured $\mathrm{PKC} \alpha$ expression and phosphorylation of FAK, which are early signaling events in both receptor pathways. Total FAK levels were similar under different conditions, but phosphorylation of FAK at Tyr-397 and $\mathrm{PKC} \alpha$ expression were decreased in cells treated with an altered FN matrix, as shown by western blot (Figure 6a). To further determine how $\mathrm{PKC} \alpha$ signals are regulated and 

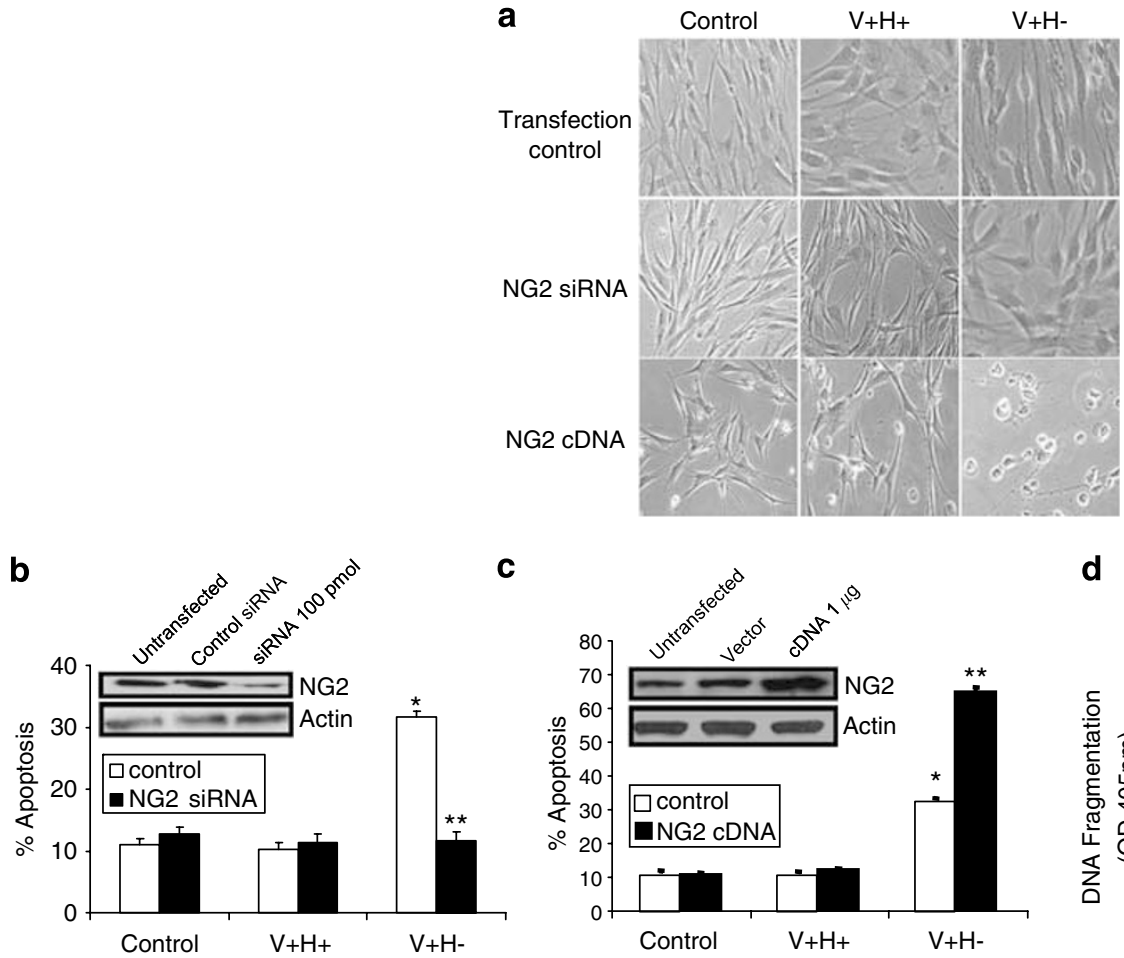

d

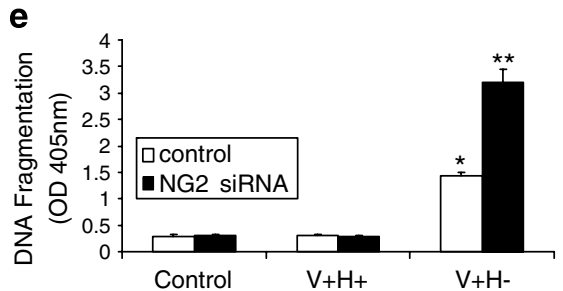

$\mathbf{f}$
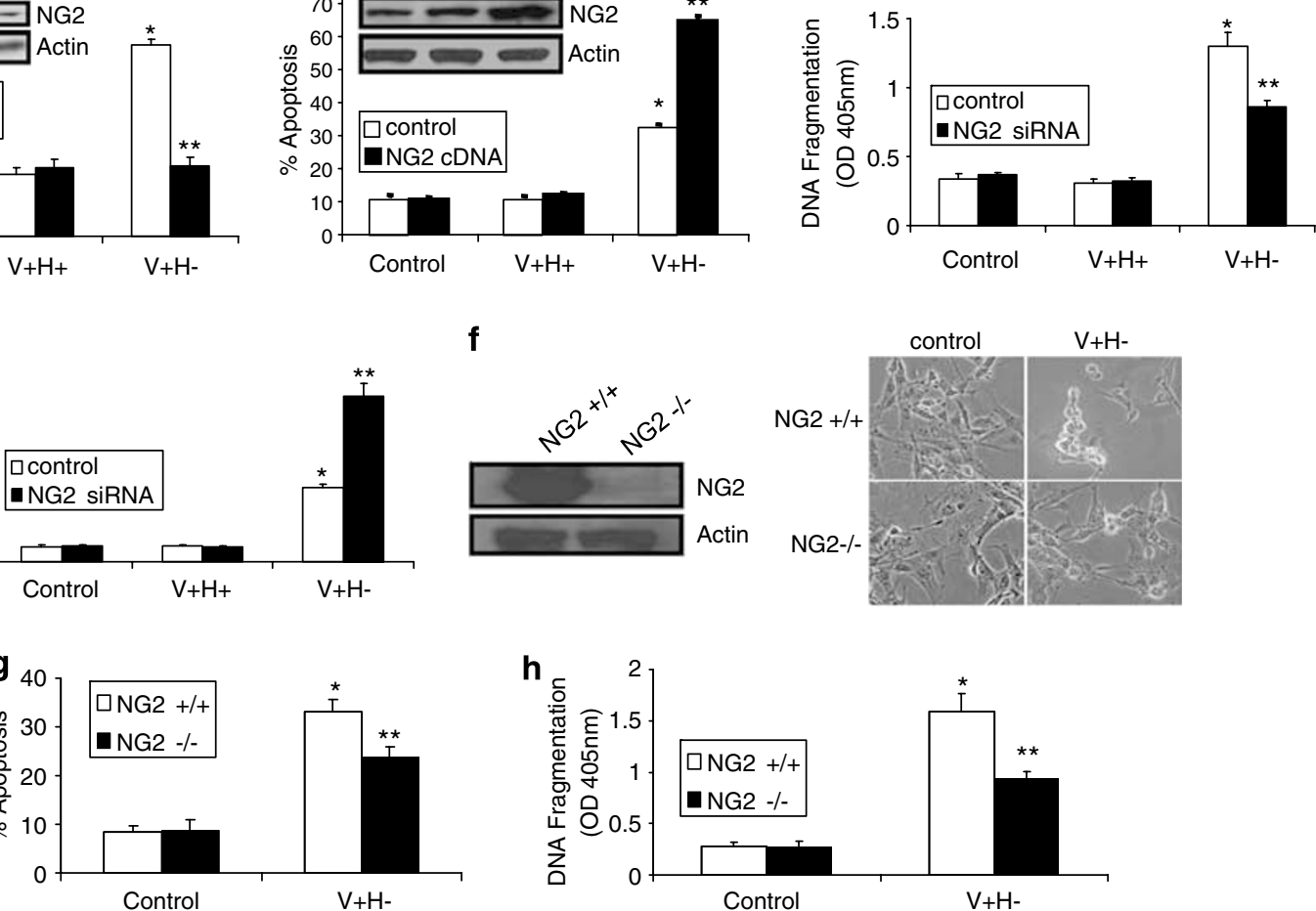

Figure 3 Anoikis induced by an altered FN matrix in primary human fibroblasts is blocked by NG2 suppression and increased by NG overexpression. (a) Phase-contrast images of cells transfected with NG2 siRNA (100 pmol), NG2 cDNA $(1 \mu \mathrm{g})$, or transfection reagent only. Cells were treated with serum-free medium (control) or the $\mathrm{V}+\mathrm{H}+\mathrm{or}$ $\mathrm{V}+\mathrm{H}-$ protein $(40 \mu \mathrm{g} / \mathrm{ml})$ for $4 \mathrm{~h}$ and photographed. Original magnification, $\times 200$. (b, c) Flow cytometric analyses of cells transfected and treated as in (a). Western blots show NG2 expression after transfection. Actin served as a loading control. (d, e) Cell death detection ELISA assays of cells transfected and treated as in (a). (f) Western blot for NG2 expression in NG2 + I+ and NG2-I- cells and phase-contrast images of NG2-null (NG2-I-) and wild-type (NG2 + I+) control fibroblast cells treated as in (a). (g) Flow cytometric analyses of NG2-null and wild-type control fibroblasts treated as in (a). (h) Cell death detection ELISA assays of NG2 null and wild-type control fibroblasts treated as in (a). Values are mean \pm S.E.M. ${ }^{\star} P<0.05$ versus control. ${ }^{* \star} P<0.05$ versus $\mathrm{V}+\mathrm{H}$ - alone

the extent to which NG2 and integrin $\alpha 4$ contribute to the regulation, we transfected cells with integrin $\alpha 4$ and NG2 cDNAs or $\alpha 4$ siRNA. Phosphorylation of FAK at Try-397 was increased by overexpression of integrin $\alpha 4$ (Figure $6 \mathrm{~b}$ ) and decreased by suppression of $\alpha 4$ (Figure $6 \mathrm{c}$ ), but $\mathrm{PKC} \alpha$ expression and total FAK protein levels were unaffected. In contrast, overexpression of NG2 decreased both $\mathrm{PKC} \alpha$ and pFAK expression (Figure 6c). Cotransfection of NG2 cDNA and integrin $\alpha 4$ siRNA did not alter $\mathrm{PKC} \alpha$ or pFAK levels more than NG2 cDNA transfection alone. Thus, NG2 negatively regulates $\mathrm{PKC} \alpha$ and pFAK levels, whereas integrin $\alpha 4$ positively regulates only pFAK levels.

Finally, to determine if $P K C \alpha$ is upstream of $p F A K$ in anoikis signaling, we assessed the effects of PKC $\alpha$ siRNA and cDNA. FAK phosphorylation was decreased by silencing $\operatorname{PKC} \alpha$ and increased by overexpressing PKC $\alpha$ (Figure 7a and b). These findings were confirmed by western blot. Total FAK protein levels were unchanged. PKC $\alpha$ overexpression rescued cells from apoptosis/anoikis (Figure 7c). 
a

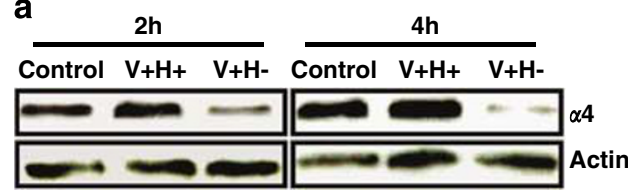

b

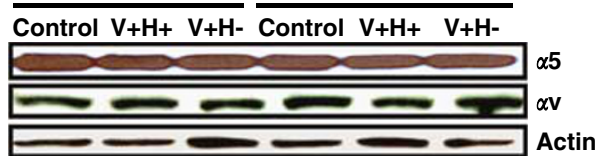

C

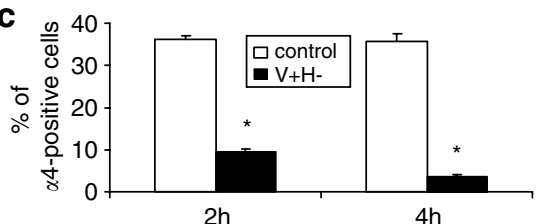

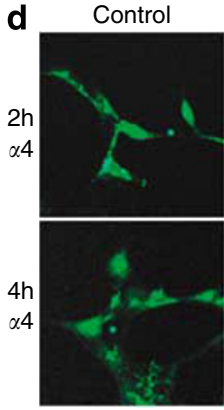

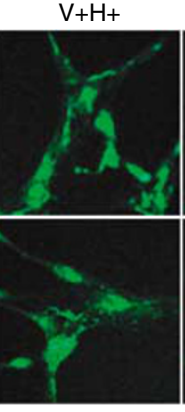

$\mathrm{V}+\mathrm{H}-$

e

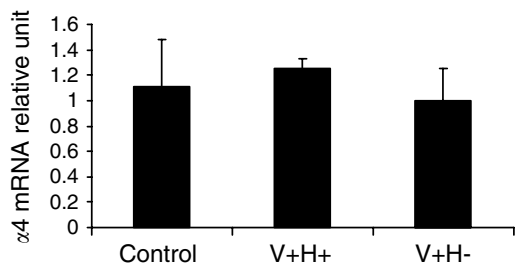

Figure 4 Integrin $\alpha 4$ expression in primary human fibroblasts is downregulated under matrix conditions that induce anoikis. (a) Western blot analysis of integrin $\alpha 4$ expression in cells treated with serum-free medium (control) or $\mathrm{V}+\mathrm{H}+$ or $\mathrm{V}+\mathrm{H}$ - protein $(40 \mu \mathrm{g} / \mathrm{ml}$ ) for 2 and $4 \mathrm{~h}$. (b) Western blot analysis of integrin $\alpha 5$ and $\alpha \mathrm{V}$ expression in cells treated as in (a). (c) Flow cytometric analysis of integrin $\alpha 4$ expression in cells incubated with $\mathrm{V}+\mathrm{H}-(40 \mu \mathrm{g} / \mathrm{ml})$ for 2 and $4 \mathrm{~h}$. (d) Immunofluorescence analysis of integrin $\alpha 4$ expression in cells treated as in (a). (e) Relative level of $\alpha 4$ mRNA expression in cells treated for $2 \mathrm{~h}$ as in (a). Values are mean $\pm S$.E.M. ${ }^{*} P<0.05$ versus control

\section{Discussion}

Previously, we showed that an altered FN matrix triggers apoptosis or anoikis in primary human fibroblasts via a signaling pathway that is regulated by decreases in FAK phosphorylation and 553 expression. ${ }^{1}$ However, the receptors involved were not known. Initial studies (Figure 1) with chondroitinase treatment suggested, since removal of CS GAGs but not HS GAGs rescued cells from anoikis, that the receptor mediating this mechanism might contain CS moieties, and thus might be a CSPG receptor. In this study, we showed that the proteoglycan NG2 and integrin $\alpha 4$ oppositely regulate this process. Treatment with an altered $\mathrm{FN}$ matrix induced anoikis in cells overexpressing NG2 but not in those overexpressing integrin $\alpha 4$. NG2 overexpression on its own induced apoptosis, whereas suppression of NG2 rescued cells from anoikis. These findings show that NG2 is a proapoptotic receptor.

NG2 regulates cell proliferation, migration, spreading, and adhesion in melanomas, gliomas, oligodendrocyte progenitors, and pericytes ${ }^{16,17,20,21}$ and increases metastasis of melanoma cells. ${ }^{20}$ It has never been implicated in apoptosis. Other proteoglycans, including syndecan-2 and syndecan-4, decorin, biglycan, CD44, and heparan sulfate proteoglycans promote or block apoptosis by modulating growth factor or integrin receptor function or by altering cell aggregation or specific signaling networks. ${ }^{22-25} \mathrm{NG2}$ also modulates growth factor receptor and integrin function to regulate a variety of cellular processes. ${ }^{17,26}$ In addition, NG2 and integrin $\alpha 4$ act as coreceptors in focal contact formation and spreading of melanoma. ${ }^{12,13}$ Here, we found that NG2 and integrin $\alpha 4$ work in opposition to modulate anoikis and that the proapoptotic activity of NG2 is increased under conditions of anoikis.

How can NG2 and $\alpha 4 \beta 1$ work in concert to mediate adhesion to intact FN but work in opposition to regulate apoptosis in response to $\mathrm{FN}$ fragments? Compared to intact
FN, FN fragments lack many of its binding sites and its threedimensional conformation, thus limiting the cellular responses the fragments can mediate. Also, NG2 decreases the adhesion of melanoma cells to CD44 monoclonal antibodies, hyaluronic acid, the C-terminal $40-\mathrm{kDa} \mathrm{FN}$ fragment, and the CS1 FN peptide, suggesting that NG2 may inhibit CD44- and $\alpha 4$-mediated events. ${ }^{20}$ Consistent with this notion, we found that NG2 overexpression promotes apoptosis by opposing integrin $\alpha 4$-mediated survival signals transmitted by pFAK. Along similar lines, NG2 and $\alpha 4$ may function as competitive inhibitors. For example, $\alpha 4$ and NG2 might bind to the same LDV hot spots on the FN fragment. ${ }^{27}$ Thus, if $\alpha 4$ is blocked, NG2-mediated adhesion increases; conversely, if NG2 is blocked, $\alpha 4$-mediated adhesion increases.

Integrin-mediated survival pathways involve the phosphorylation and activation of intracellular tyrosine kinases, such as FAK $^{28,29}$ FAK itself may be modulated by PKC signaling, as in focal adhesion formation and in regulating downstream signals from FAK, which are important for cell survival, cell spreading, and cell migration. ${ }^{30} \mathrm{NG} 2$ interacts directly with both $\mathrm{PKC} \alpha$ and $\mathrm{ERK}^{17}$ and appears to regulate FAK and ERK activation; ${ }^{13,26,31}$ however, it was not known whether NG2-mediated signal transduction pathways lead to apoptosis or anoikis. We found anoikis signals mediated by an altered FN matrix and transmitted by NG2 led to decreases in PKC $\alpha$ and pFAK levels. Overexpression of integrin $\alpha 4$ increased pFAK (Tyr-397), but not PKC $\alpha$ levels, indicating that the pFAK survival signal is mediated independently of PKC $\alpha$. Overexpression of NG2 decreased both PKC $\alpha$ levels and phosphorylation of FAK, while suppression of PKC $\alpha$ decreased FAK phosphorylation, indicating that NG2 regulates FAK phosphorylation through PKC $\alpha$ in fibroblasts. Thus, NG2, but not integrin $\alpha 4$, regulates anoikis of fibroblasts via changes in FAK phosphorylation through a PKC $\alpha$-dependent mechanism. 


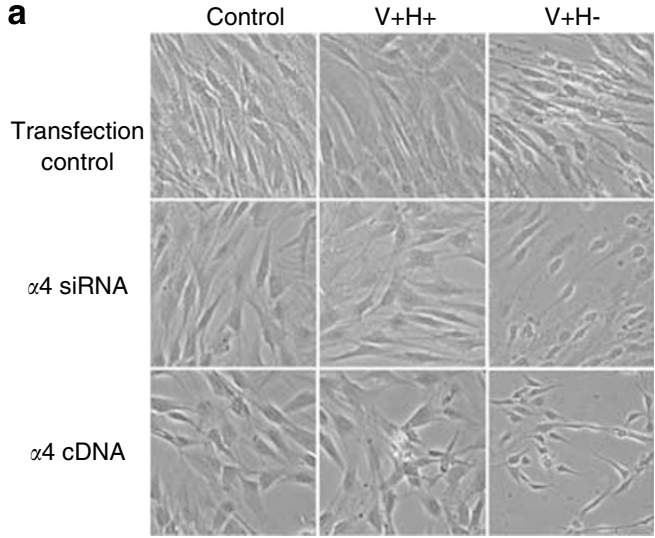

b
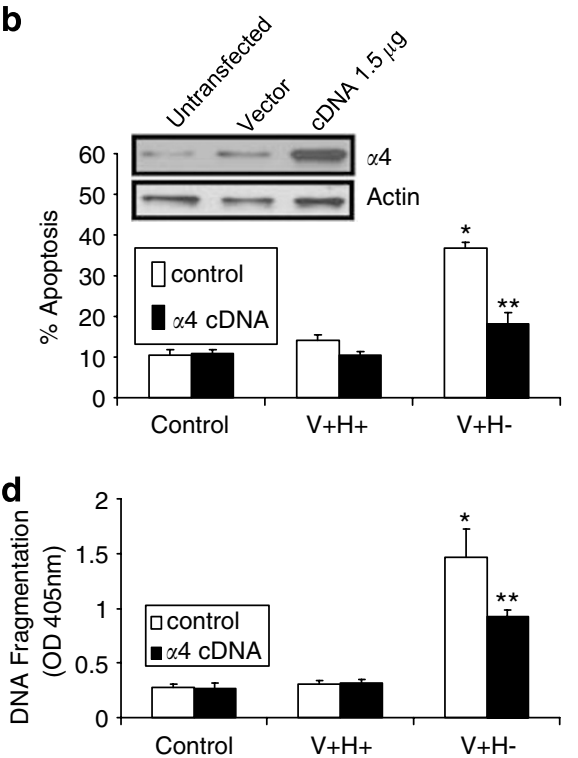

c
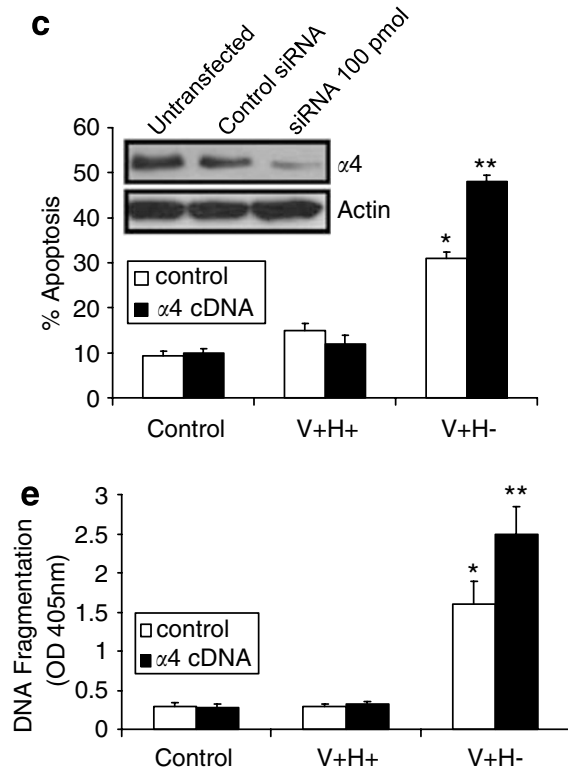

Figure 5 Overexpression of integrin $\alpha 4$ rescues primary human fibroblasts from anoikis induced by an altered FN matrix. (a) Phase-contrast images of cells transfected with $\alpha 4$ siRNA (100 pmol), integrin $\alpha 4 \mathrm{cDNA}(1.5 \mu \mathrm{g})$, or transfection reagent. Cells were then treated with control serum-free medium (control) or with $\mathrm{V}+\mathrm{H}+$ or $\mathrm{V}+\mathrm{H}-$ protein $(40 \mu \mathrm{g} / \mathrm{ml})$ for $4 \mathrm{~h}$ and photographed. Original magnification, $\times 200$. (b, c) Flow cytometric analyses of apoptosis. Cells were transfected and treated as in (a). Western blots show the level of $\alpha 4$ expression after transfection. Actin served as a loading control. (d, e) Cell death detection ELISA assays of cells transfected and treated as in (a). Values are mean \pm S.E.M. ${ }^{\star} P<0.05$ versus control. ${ }^{\star \star} P<0.05$ versus $\mathrm{V}+\mathrm{H}-$ alone

NG2 might regulate anoikis signaling in a PKC $\alpha$-dependent manner by binding to $\mathrm{PKC} \alpha^{17}$ or modulating its degradation or transcription. Future experimentation will help clarify this situation. Another important area of investigation may include determination of the phosphorylation status of NG2 under control and proapoptotic conditions. Phosphorylation of NG2 at Thr-2256 or Thr-2314 controls its localization to specific membrane microdomains, along with its ability to activate $\beta 1$ integrins and downstream signaling pathways to selectively stimulate cell motility or proliferation. ${ }^{17}$ It seems possible that NG2's phosphorylation state and localization pattern influence its ability to direct integrin-mediated anoikis signaling in response to altered matrix composition.

\section{Materials and Methods}

Cell culture. Primary human ligament fibroblasts were obtained as described. ${ }^{1}$ Fibroblasts were maintained in $\alpha$-minimal essential medium containing $10 \%$ fetal bovine serum and $1 \%$ penicillin-streptomycin-fungizone. NG2-null fibroblasts and wild-type control fibroblasts ${ }^{26}$ were maintained in Dulbecco's modified Eagle's medium containing $10 \%$ fetal bovine serum and $1 \%$ penicillin-streptomycin.

Altered FN matrix/recombinant FN proteins. Two recombinant FN proteins, $\mathrm{V}+\mathrm{H}+$ (control) and $\mathrm{V}+\mathrm{H}-$ (altered matrix), were prepared as described. ${ }^{32}$ The $\mathrm{V}+\mathrm{H}$ - fragment is comparable to the $40 \mathrm{kDa}$ naturally occurring proteolytic fragment found in diseased patient fluids. ${ }^{33,34}$ Both fragments rely on interactions with the heparin-binding domain and $\mathrm{V}$ region of $\mathrm{FN}$.

Plasmids and siRNAs. NG2 cDNA (pcDNAamp/NG2) has been described. ${ }^{25,35}$ NG2 siRNA and mutant control NG2 siRNA were transcribed in vitro with chemically synthesized DNA oligonucleotides and T7 RNA polymerase as described ${ }^{36}$ Synthesis and annealing were confirmed by non-denaturing PAGE. Human NG2 was targeted with siRNA (5'-GUGGACCAGUACCCUACGG-3') corresponding to human melanoma-associated CSPG (cspg4). A non-functional mutant control siRNA with four consecutive nucleotide mismatches served as a control ( $5^{\prime}$-GUAGAUCAAUUGGGUACACUU-3'). Only the sense strand is shown in each case. NG2 suppression was also confirmed with commercially available siRNA and cognate Stealth RNAi negative control (Invitrogen, Carlsbad, CA, USA). Integrin $\alpha 4$ cDNA was from the American Type Culture Collection (Manassas, VA, USA). Integrin $\alpha 4$ siRNA was from Ambion (Austin, TX, USA). PKC $\alpha$ cDNA was a gift from 

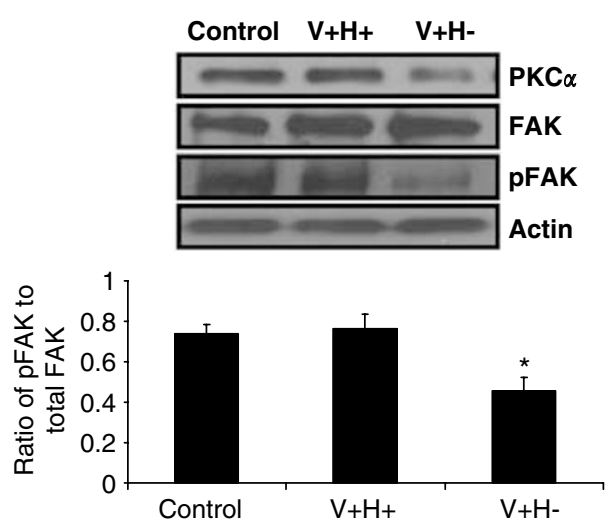

C b
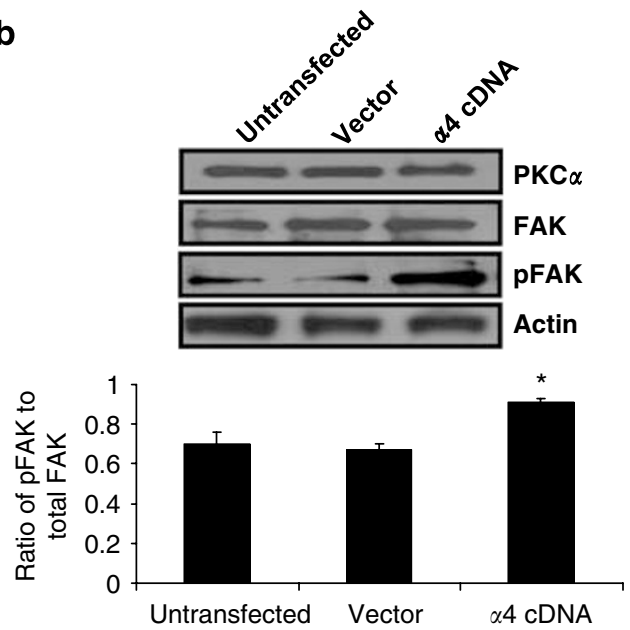
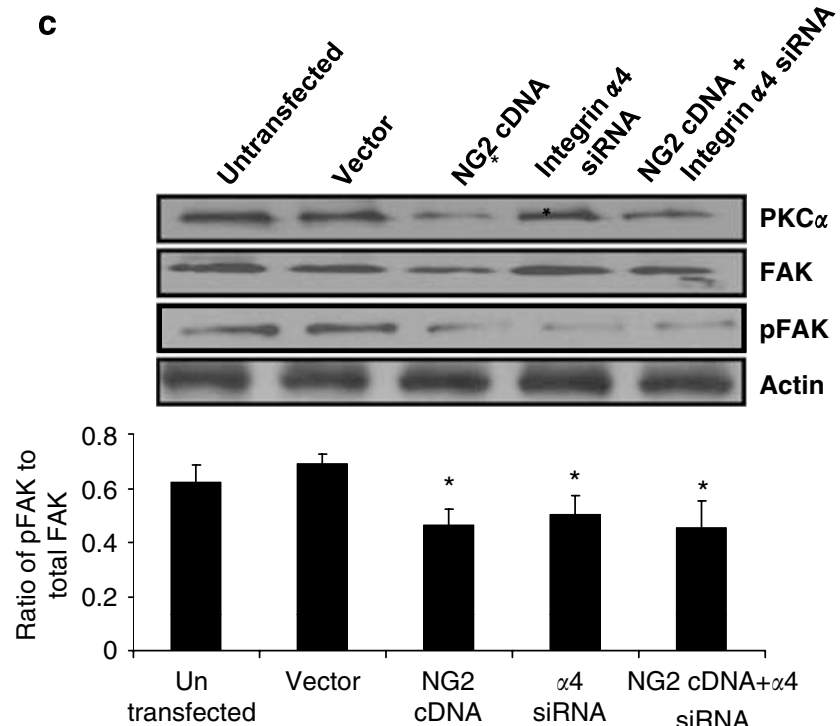

Figure 6 NG2 and integrin $\alpha 4$ regulate FAK phosphorylation by PKC $\alpha$-dependent and -independent mechanisms in human primary fibroblasts. (a, b) Western blot analysis of PKC $\alpha, F A K$, and pFAK expression in cells treated with serum-free medium (control) or V $+\mathrm{H}+$ or V $+\mathrm{H}-$ protein $(40 \mu \mathrm{g} / \mathrm{ml})$ for $2 \mathrm{~h}(\mathrm{a})$, transfected with $1.5 \mu \mathrm{g}$ of integrin $\alpha 4 \mathrm{cDNA}$ (b) or transfected with vector control, $1 \mu \mathrm{g}$ of NG2 cDNA (lane 3), $100 \mathrm{pmol}$ of integrin $\alpha 4$ siRNA (lane 4), or both (lane 5) (c). (a-c) The ratios of phosphorylated to total FAK were quantified by densitometric analysis. Values are mean \pm S.E.M. ${ }^{*} P<0.05$ versus control

Dr. Silvio Gutkind (NIH, Bethesda, MA, USA). PKC $\alpha$ siRNA was from Santa Cruz Biotechnology (Santa Cruz, CA, USA).

Nuclear staining. Nuclear staining of DNA with a fluorescent groove-binding probe for DNA (DAPI; Sigma, St. Louis, MO, USA) was used as described. ${ }^{2}$

Apoptosis assay. Cells were treated with recombinant FN proteins, harvested, and suspended in enzyme-free dissociation buffer (Invitrogen); in some experiments, cells were pretreated with chondroitinase ABC (Sigma; $0.2 \mathrm{U}$ per well), heparinase III (Sigma; $0.1 \mathrm{U}$ per well), or both. Cell suspensions were stained with annexin V conjugated to FITC (BD Biosciences, Franklin Lakes, NJ, USA). The percentage of apoptotic cells was determined with a FACSDiVA Cell Sorter (Becton Dickinson, Franklin Lakes, NJ, USA).

Apoptotic cell death detection by ELISA. Histone-associated DNA fragments (mono- and oligonucleosomes) in the cytoplasm were determined by Cell

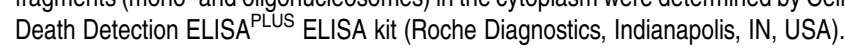
Briefly, the lysates from floating and adherent cells were placed in a streptavidincoated microplate and incubated with a mixture of anti-histone-biotin and anti-DNA peroxidase. After addition of peroxidase substrate (ABTS), the absorbance was measured at $405 \mathrm{~nm}$.
Analysis of NG2 and integrin $\alpha \mathbf{4}$ by flow cytometry. NG2 and integrin $\alpha 4$ expression were quantified by flow cytometry. After treatment with recombinant FN proteins, cells were washed with PBS and pelleted by centrifugation. Parallel samples were incubated with B5 mouse anti-NG2 antibody (American Type Culture Collection) or rabbit anti-integrin $\alpha 4$ antibody (Santa Cruz Biotechnology) at $4{ }^{\circ} \mathrm{C}$ for $2 \mathrm{~h}$. Cells were washed in PBS, pelleted by centrifugation, and incubated with a second FITC-conjugated antibody for $30 \mathrm{~min}$ in the dark. Samples were stored in the dark for $20 \mathrm{~min}$ before analysis.

Immunofluorescence. Cells were incubated with B5 mouse anti-NG2 and rabbit anti-integrin $\alpha 4$ antibody (Chemicon, Temecula, CA, USA) and prepared for immunofluorescence as described. ${ }^{2}$

Western blotting. Samples were electrophoretically resolved by standard SDS-PAGE and western blotting as previously described. ${ }^{4}$ Samples for NG2 (proteoglycan) detection were pretreated with $0.2 \mathrm{U}$ of chondroitinase $\mathrm{ABC}$ for $1 \mathrm{~h}$ at $37^{\circ} \mathrm{C}$. NG2 was detected with B5 monoclonal antibody. Polyclonal anti-integrin $\alpha 4$, $\alpha 5$, CD44, and syndecan-2 were from Santa Cruz Biotechnology. Polyclonal antiintegrin $\alpha v$ was from Chemicon. Polyclonal anti syndecan-4 was from Biovision (Mountain View, CA, USA). Densitometric analysis of bands of exposed films was performed using NIH Image software. 
a

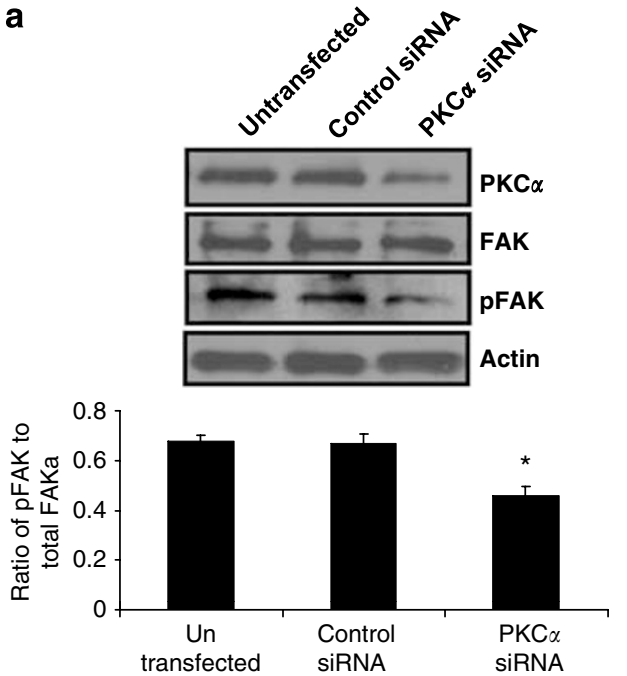

b
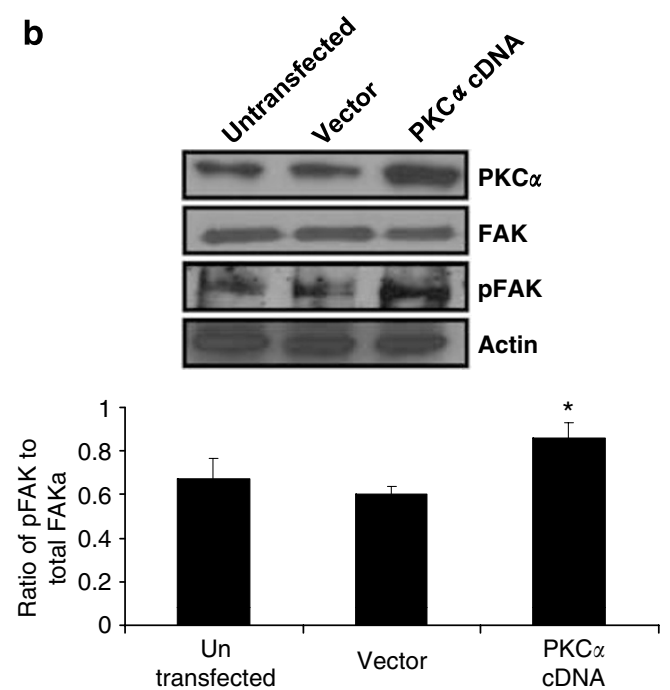

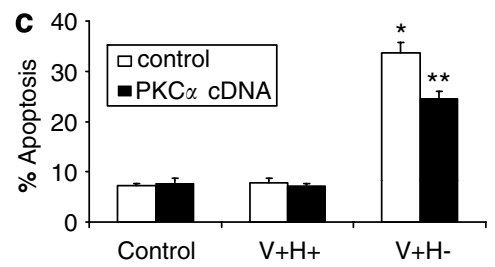

Figure 7 PKC $\alpha$ is upstream of pFAK and overexpression of PKC $\alpha$ rescues cells from anoikis induced by an altered FN matrix. (a) Western blot analyses of PKC $\alpha$, FAK, and pFAK in untransfected cells and cells transfected with control siRNA or $100 \mathrm{pmol}$ of PKC $\alpha$ siRNA. (b) Western blot analyses of PKC $\alpha$, FAK, and pFAK in control untransfected cells and cells transfected with vector control or $1 \mu \mathrm{g}$ of PKC $\alpha$ cDNA. (a-b) The ratios of phosphorylated to total FAK were quantified by densitometric analysis. (c) Flow cytometric analysis of apoptosis in control transfected cells and cells transfected with $1 \mu \mathrm{g}$ of PKC $\alpha \mathrm{CDNA}$ and treated with serum-free medium (control) or with $\mathrm{V}+\mathrm{H}+$ protein or $\mathrm{V}+\mathrm{H}-$ protein $(40 \mu \mathrm{g} / \mathrm{ml})$ for $2 \mathrm{~h}$. Values are mean \pm S.E.M. ${ }^{*} P<0.05$ versus control. ${ }^{\star \star} P<0.05$ versus $\mathrm{V}+\mathrm{H}-$ alone

DNA transfection in fibroblast cells. At $60-80 \%$ confluency, cells in sixwell tissue culture plates were transiently transfected with cDNA or vector control using Lipofectamine 2000 (Invitrogen) for $6 \mathrm{~h}$, washed, and incubated with $\alpha \mathrm{MEM} /$ $10 \%$ FBS for $36 \mathrm{~h}$. Cells were then treated with recombinant FN proteins in serumfree medium for experiments. Transfection efficiency was assessed by western blot of cell extracts 24,36 , and $48 \mathrm{~h}$ after transfection.

RNAi. Cells were transiently transfected with siRNA, non-functional control NG2 siRNA, or Stealth RNAi Negative Control (Invitrogen) using Lipofectamine 2000. Cells were then treated with recombinant FN proteins in serum-free medium for experiments. To monitor gene silencing, cell extracts were assessed by western blot 24,36 , and $48 \mathrm{~h}$ after transfection.

Real-time RT-PCR. mRNA expression of NG2 and integrin $\alpha 4$ was measured by two-step quantitative RT-PCR. After treatment with FN proteins, total RNA was extracted with the RNeasy Mini Kit (Qiagen, Valencia, CA, USA). First-strand cDNAs were synthesized with an anchored oligo(dT) primer (Invitrogen). Real-time PCR was performed on an Applied Biosystems 7500 Real Time PCR System. Target gene expression was normalized to glyceraldehyde-3-phosphate dehydrogenase (GAPDH). The primers were from Applied Biosystems (Foster City, CA, USA). The sequences were NM_001897.3 (human NG2), NM_000885.4 (human integrin $\alpha 4$ ), and NM_002046.3 (human GAPDH).

Statistical analysis. Data were analyzed by a two-way ANOVA and the intergroup differences were determined by Fisher's PLSD test. Innergroup differences were analyzed by an unpaired Student's $t$-test. $P<0.05$ was considered significant. Values are mean \pm S.E.M. All experiments were performed in triplicate.

Acknowledgements. We thank Dr. Silvio Gutkind (NIH) for the PKC $\alpha$ cDNA, Paul W Johnson for recombinant FN proteins, and Stephen Ordway for editorial assistance. This study was supported by NIH grants R01-DE13725 (YLK) and R01CA95287 (WBS).

1. Kapila YL, Wang S, Johnson PW. Mutations in the heparin binding domain of fibronectin in cooperation with the $\mathrm{V}$ region induce decreases in pp125(FAK) levels plus proteoglycan mediated apoptosis via caspases. J Biol Chem 1999; 274: 30906-30913.

2. Tafolla $E$, Wang $S$, Wong $B$, Leong J, Kapila YL. JNK1 and JNK2 oppositely regulate $p 53$ in signaling linked to apoptosis triggered by an altered fibronectin matrix: JNK links FAK and p53. J Biol Chem 2005; 280: 19992-19999.

3. Kapila YL, Wang S, Dazin P, Tafolla E, Mass MJ. The heparin-binding domain and V region of fibronectin regulate apoptosis by suppression of p53 and c-myc in human primary cells. J Biol Chem 2002; 277: 8482-8491.

4. Frisch SM, Vuori K, Ruoslahti E, Chan-Hui PY. Control of adhesion-dependent cell survival by focal adhesion kinase. J Cell Biol 1996; 134: 793-799.

5. Ilic D, Almeida EA, Schlaepfer DD, Dazin P, Aizawa S, Damsky CH. Extracellular matrix survival signals transduced by focal adhesion kinase suppress p53-mediated apoptosis. J Cell Biol 1998; 143: 547-560

6. Hungerford JE, Compton MT, Matter ML, Hoffstrom BG, Otey CA. Inhibition of pp125FAK in cultured fibroblasts results in apoptosis. J Cell Biol 1996; 135: 1383-1390.

7. Vuori K, Ruoslahti E. Activation of protein kinase $\mathrm{C}$ precedes alpha 5 beta 1 integrinmediated cell spreading on fibronectin. J Biol Chem 1993; 268: 21459-21462.

8. Schlaepfer DD, Hanks SK, Hunter T, van der Geer P. Integrin-mediated signal transduction linked to Ras pathway by GRB2 binding to focal adhesion kinase. Nature 1994; $\mathbf{3 7 2}$ : 786-791.

9. Haimovich $B$, Kaneshiki N, Ji P. Protein kinase $C$ regulates tyrosine phosphorylation of pp125FAK in platelets adherent to fibrinogen. Blood 1996; 87: 152-161.

10. Woods A, Couchman JR. Protein kinase $C$ involvement in focal adhesion formation. J Cell Sci 1992; 101 (Pt 2): 277-290.

11. Clark EA, Brugge JS. Integrins and signal transduction pathways: the road taken. Science 1995; 268: 233-239

12. lida J, Meijne AM, Spiro RC, Roos E, Furcht LT, McCarthy JB. Spreading and focal contact formation of human melanoma cells in response to the stimulation of both melanomaassociated proteoglycan (NG2) and alpha 4 beta 1 integrin. Cancer Res 1995; 55 2177-2185. 
13. Yang J, Price MA, Neudauer $\mathrm{CL}$, Wilson $\mathrm{C}$, Ferrone $\mathrm{S}$, Xia $\mathrm{H}$ et al. Melanoma chondroitin sulfate proteoglycan enhances FAK and ERK activation by distinct mechanisms. J Cell Biol 2004; 165: 881-891.

14. Stallcup WB. The NG2 proteoglycan: past insights and future prospects. $J$ Neurocyto 2002; 31: 423-435.

15. Midwood KS, Salter DM. Expression of NG2/human melanoma proteoglycan in human adult articular chondrocytes. Osteoarthr Cartil 1998; 6: 297-305.

16. Tillet $E$, Gential B, Garrone R, Stallcup WB. NG2 proteoglycan mediates beta1 integrinindependent cell adhesion and spreading on collagen VI. J Cell Biochem 2002; 86: 726-736.

17. Makagiansar IT, Williams S, Mustelin T, Stallcup WB. Differential phosphorylation of NG2 proteoglycan by ERK and PKCalpha helps balance cell proliferation and migration. $J$ Cell Biol 2007; 178: 155-165.

18. Barritt DS, Pearn MT, Zisch AH, Lee SS, Javier RT, Pasquale EB et al. The multi-PDZ domain protein MUPP1 is a cytoplasmic ligand for the membrane-spanning proteoglycan NG2. J Cell Biochem 2000; 79: 213-224.

19. Stegmuller J, Werner $\mathrm{H}$, Nave KA, Trotter J. The proteoglycan NG2 is complexed with alpha-amino-3-hydroxy-5-methyl-4-isoxazolepropionic acid (AMPA) receptors by the PDZ glutamate receptor interaction protein (GRIP) in glial progenitor cells. Implications for glial-neuronal signaling. J Biol Chem 2003; 278: 3590-3598.

20. Burg MA, Grako KA, Stallcup WB. Expression of the NG2 proteoglycan enhances the growth and metastatic properties of melanoma cells. J Cell Physiol 1998; 177: 299-312.

21. Ozerdem U, Stallcup WB. Pathological angiogenesis is reduced by targeting pericytes via the NG2 proteoglycan. Angiogenesis 2004; 7: 269-276.

22. Schaefer L, Macakova K, Raslik I, Micegova M, Grone HJ, Schonherr E et al. Absence of decorin adversely influences tubulointerstitial fibrosis of the obstructed kidney by enhanced apoptosis and increased inflammatory reaction. Am J Pathol 2002; 160: $1181-1191$.

23. Modrowski D, Orosco A, Thevenard J, Fromigue O, Marie PJ. Syndecan-2 overexpression induces osteosarcoma cell apoptosis: implication of syndecan-2 cytoplasmic domain and JNK signaling. Bone 2005; 37: 180-189.
24. Bi $\mathrm{Y}$, Stuelten $\mathrm{CH}$, Kilts $\mathrm{T}$, Wadhwa $\mathrm{S}$, lozzo RV, Robey $\mathrm{PG}$ et al. Extracellular matrix proteoglycans control the fate of bone marrow stromal cells. J Biol Chem 2005; 280: 30481-30489.

25. Seidler DG, Goldoni S, Agnew C, Cardi C, Thakur ML, Owens RT et al. Decorin protein core inhibits in vivo cancer growth and metabolism by hindering epidermal growth factor receptor function and triggering apoptosis via caspase-3 activation. J Biol Chem 2006; 281 : 26408-26418.

26. Grako KA, Ochiya T, Barritt D, Nishiyama A, Stallcup WB. PDGF (alpha)-receptor is unresponsive to PDGF-AA in aortic smooth muscle cells from the NG2 knockout mouse. J Cell Sci 1999; 112 (Pt 6): 905-915.

27. Wayner EA, Kovach NL. Activation-dependent recognition by hematopoietic cells of the LDV sequence in the V region of fibronectin. J Cell Biol 1992; 116: 489-497.

28. Parsons JT. Focal adhesion kinase: the first ten years. J Cell Sci 2003; 116: 1409-1416.

29. Mitra SK, Schlaepfer DD. Integrin-regulated FAK-Src signaling in normal and cancer cells. Curr Opin Cell Biol 2006; 18: 516-523.

30. Lewis JM, Cheresh DA, Schwartz MA. Protein kinase $C$ regulates alpha $v$ beta 5dependent cytoskeletal associations and focal adhesion kinase phosphorylation. J Cell Biol 1996; 134: 1323-1332.

31. Chang CC, Campoli M, Luo W, Zhao W, Zaenker KS, Ferrone S. Immunotherapy of melanoma targeting human high molecular weight melanoma-associated antigen: potential role of nonimmunological mechanisms. Ann NY Acad Sci 2004; 1028: 340-350.

32. Kapila YL, Niu J, Johnson PW. The high affinity heparin-binding domain and the $V$ region of fibronectin mediate invasion of human oral squamous cell carcinoma cells in vitro. J Biol Chem 1997; 272: 18932-18938.

33. Huynh QN, Wang S, Tafolla E, Gansky SA, Kapila S, Armitage GC et al. Specific fibronectin fragments as markers of periodontal disease status. J Periodontol 2002; 73: 1101-1110.

34. Dai R, Iwama A, Wang S, Kapila YL. Disease-associated fibronectin matrix fragments trigger anoikis of human primary ligament cells: p53 and c-myc are suppressed. Apoptosis 2005; 10: 503-512.

35. Nishiyama A, Stallcup WB. Expression of NG2 proteoglycan causes retention of type $\mathrm{VI}$ collagen on the cell surface. Mol Biol Cell 1993; 4: 1097-1108.

36. Leirdal M, Sioud M. Gene silencing in mammalian cells by preformed small RNA duplexes. Biochem Biophys Res Commun 2002; 295: 744-748. 